\title{
Amphibians of Serra Bonita, southern Bahia: a new hotpoint within Brazil's Atlantic Forest hotspot
}

\author{
Iuri Ribeiro Dias ${ }^{1,2}$, Tadeu Teixeira Medeiros ${ }^{3}$, \\ Marcos Ferreira Vila Nova', Mirco Solé, ${ }^{\prime 2}$
}

I Departamento de Ciências Biológicas, Universidade Estadual de Santa Cruz, Rodovia Jorge Amado, km, 16, 45662-900 Ilhéus, Bahia, Brasil 2 Graduate Program in Applied Zoology, Universidade Estadual de Santa Cruz, Rodovia Jorge Amado, km 16, $45662-900$ Ilhéus, Bahia, Brasil 3 Conselho de Curadores das Coleçöes Cientificas, Universidade Estadual de Santa Cruz, Rodovia Jorge Amado, km 16, 45662-900 Ilhéus, Bahia, Brasil

Corresponding author: Iuri Ribeiro Dias (iurirdias@hotmail.com)

Academic editor: F. Andreone | Received 12 March 2014 | Accepted 12 September 2014 | Published 22 October 2014

http://zoobank.org/4BE3466B-3666-4012-966D-350CA6551E15

Citation: Dias IR, Medeiros TT, Nova MFV, Solé M (2014) Amphibians of Serra Bonita, southern Bahia: a new hotpoint within Brazil's Atlantic Forest hotspot. ZooKeys 449: 105-130. doi: 10.3897/zookeys.449.7494

\begin{abstract}
We studied the amphibian community of the Private Reserve of Natural Heritage (RPPN) Serra Bonita, an area of $20 \mathrm{~km}^{2}$ with steep altitudinal gradients (200-950 m a.s.l.) located in the municipalities of Camacan and Pau-Brasil, southern Bahia State, Brazil. Data were obtained at 38 sampling sites (including ponds and transects within the forest and in streams), through active and visual and acoustic searches, pitfall traps, and opportunistic encounters. We recorded 80 amphibian species distributed in 15 families: Aromobatidae (1), Brachycephalidae (3), Bufonidae (4), Centrolenidae (2), Ceratophryidae (1), Craugastoridae (7), Eleutherodactylidae (2), Hemiphractidae (2), Hylidae (42), Hylodidae (1), Leptodactylidae (7), Microhylidae (3), Siphonopidae (1), Odontophrynidae (3) and Pipidae (1). Species richness was positively correlated with monthly rainfall. Near $36 \%$ of the species were found in strictly forest environments, $15 \%$ are endemic to Bahia State and $77.2 \%$ are endemic to the Atlantic Forest biome. The large species diversity of this small area, the high degree of endemism and the taxonomic and biogeographic significance turn the Serra Bonita mountain into a hotpoint for amphibians within Brazil's Atlantic Forest hotspot.
\end{abstract}

\section{Resumo}

Neste trabalho, estudamos a comunidade de anfíbios da Reserva Particular do Patrimônio Natural (RPPN) Serra Bonita que possui área de $20 \mathrm{~km}^{2}$ com gradientes altitudinais abruptos (200-950 m acima do nível do mar), localizada nos municípios de Camacan e Pau Brasil, sul do Estado da Bahia, Brasil. Os dados foram obtidos em 38 diferentes sítios amostrais (incluindo poças, transectos no interior da floresta e em riachos)

Copyright luri Ribeiro Dias et al. This is an open access article distributed under the terms of the Creative Commons Attribution License (CC BY 4.0), which permits unrestricted use, distribution, and reproduction in any medium, provided the original author and source are credited. 
através de procura ativa visual e acústica, armadilhas de interceptação e queda e por encontros oportunísticos. Foram registradas 80 espécies de anfíbios distribuídas em 15 famílias: Aromobatidae (1), Brachycephalidae (3), Bufonidae (4), Centrolenidae (2), Ceratophryidae (1), Craugastoridae (7), Eleutherodactylidae (2), Hemiphractidae (2), Hylidae (42), Hylodidae (1), Leptodactylidae (7), Microhylidae (3), Siphonopidae (1), Odontophrynidae (3) e Pipidae (1). A riqueza foi positivamente correlacionada com a precipitaçáo mensal. Aproximadamente $36 \%$ das espécies foram encontradas em ambientes estritamente florestais, 15\% são endêmicas do Estado da Bahia e 77.2\% são endêmicas do bioma Mata Atlântica. A alta diversidade de espécies desta pequena área, o elevado endemismo e a relevância taxonômica e biogeográfica tornam a Serra Bonita um 'hotpoint' para anfíbios no 'hotspot' da Mata Atlântica.

\section{Keywords}

Anura, Mountain, Biodiversity, species distribution, species richness

\section{Introduction}

Among vertebrates, amphibians are considered the most threatened group on the planet (Hoffmann et al. 2010). Near a third of the world's amphibian species are endangered (Stuart et al. 2004). The main threats to the group are loss and fragmentation of habitat (Young et al. 2004, Becker et al. 2007, Loyola et al. 2007), climate change (Pounds et al. 2006, Blaustein and Johnson 2003), introduction of exotic species (Kats and Ferrer 2003) and diseases (Lips et al. 2003).

Despite Brazil showing the highest worldwide diversity of amphibians (Segalla et al. 2012), it does not rank within the first positions in the number of endangered species. This is probably due to the lack of data regarding most Brazilian species, once the country is also the world leader in species classified as Data Deficient (DD) - near 25\% of the assessed species (IUCN 2008). Many of this species could be facing extinction but are not in the scope of conservation actions, since we cannot determine the major threats to their populations due to the absence of adequate sampling along the Brazilian territory (Trindade-Filho et al. 2012, Verdade et al. 2012, Campos et al. 2013, Morais et al. 2013).

Brazil's Atlantic Forest is one of the five most important global hotspots of biodiversity (Myers et al. 2000). It harbors more than half of the country's amphibians (Haddad et al. 2013) and exhibits high endemism rates (> 85\% of species) for this taxonomic group (Cruz and Feio 2007). Such high biodiversity is faced with the current situation of the biome, with fewer than $11.7 \%$ of its original area remaining and only $1 \%$ of its total area being legally protected (Ribeiro et al. 2009). In addition, the number of amphibian species is expected to decline within the Atlantic Forest network of protected areas due to climate change, according to projections made by Lemes et al. (2014).

Given the devastation of the Atlantic Forest biome, where more than half of the Brazilian amphibians live, coupled with the lack of information to assess the conservation status of many species, primary studies are urgently needed to overcome these shortfalls.

The central region of the Atlantic Forest - including the south of Bahia - was deemed as a zone of climatic stability during the Quaternary glaciations and was the 
greatest refuge for amphibians in the Atlantic Forest during that period (Carnaval et al. 2009). This region is regarded as an important center of diversification and endemism for plants (Thomas et al. 1998) and different groups of animals (Haffer 1974, Brown 1991, Bencke et al. 2006).

In a comparison of the diversity of trees from different tropical forests in the world, a forest remnant of southern Bahia was amongst those with the greatest richness and was thereby considered a hotpoint within the Atlantic Forest hotspot (Martini et al. 2007). The identification of areas with large numbers of endemic species and species diversity within the major global hotspots assists in planning conservation actions aimed at smaller areas that can be more easily managed and protected than larger ones (Murray-Smith et al. 2008).

The study of the species richness and species composition in a given area is essential to know the functional structure of biological communities, as well as to understand the dynamics between fragments, serving as an instrument that contributes in decisions relative to species conservation (Droege et al. 1998, Haddad 1998). As highlighted by Verdade et al. (2012), further investments in basic research - in particular regarding the collection of field data during inventories of fauna and taxonomic reviews - are necessary to better understand the extraordinary biodiversity of Brazilian amphibians as to properly assess their conservation status and help design conservation strategies for this taxonomic group.

There is a huge lack of information about the occurrence of amphibians in the State of Bahia, Brazil. This can be noticed by the frequent publication of notes regarding the increased distribution of species in the state (e.g. Orrico 2010, Dias et al. 2010, Camurugi et al. 2010, Dias et al. 2011) and the large number of new species described over the last years (e.g. Napoli et al. 2011a, Lourenço-de-Moraes et al. 2012, Teixeira-Jr et al. 2013, Caramaschi et al. 2013). Furthermore, only eleven scientific articles contain lists of amphibian species in the state (Silvano and Pimenta 2003, Juncá 2005, Juncá 2006, Bastazini et al. 2007, Protázio et al. 2009, Valdujo et al. 2009, Camurugi et al. 2010, Xavier and Napoli 2011, Valdujo et al. 2011, Garda et al. 2013, Lantyer-Silva et al. 2013). Most of the available information regarding amphibians of southern Bahia are results of the work of Silvano and Pimenta (2003). Despite the fact that a small sampling effort was applied (about four nights at each fragment), a considerable number of fragments were sampled in 19 localities and a total of 92 species were found.

Due to difficult access or the lack of suitability for agricultural purposes, most forest remnants that exist outside protected areas in southern Bahia are situated on slopes or mountain tops (Thomas et al. 1998, 2008). From the scientific point of view, though, these places are still little explored even considering areas of utmost biological importance (Martinelli 2007). As for the montane areas, the few existing data exhibit a high diversity of species (Amorim et al. 2005, 2009, Matos et al. 2010, Rocha and Amorim 2012), many of which endemic and new to science (e.g. Amorim and Leme 2009, Napoli et al. 2011a, Machado et al. 2013, Teixeira-Jr et al. 2013).

Accordingly, the objective of this study was to inventory the amphibians of the Private Reserve of Natural Heritage (RPPN) Serra Bonita, a montane area covered by 
Tropical Rainforest in southern Bahia State, Brazil. Our data reveal that Serra Bonita is one of the amphibian habitats with the greatest diversity in the world, a hotpoint within the Atlantic Forest hotspot for this taxonomic group.

\section{Materials and methods}

\section{Study area}

The Serra Bonita Reserve (Figure 1) is located in the municipalities of Camacan and Pau-Brasil, Bahia State, Brazil $\left(15^{\circ} 23^{\prime} \mathrm{S}, 39^{\circ} 33^{\prime} \mathrm{W}\right)$. The region is known as the "South Coast" and is located about $130 \mathrm{~km}$ from the city of Ilhéus and $526 \mathrm{~km}$ from the state capital - Salvador. Under a free lease agreement, the Uiraçu Institute administers around $20 \mathrm{~km}^{2}$ from which 12 were transformed into a Private Reserve of Natural Heritage (PRNP or RPPN) located within the Central Corridor of the Atlantic Forest (Instituto Uiraçu 2009).

The area comprises steep altitudinal gradients (200-950 m a.s.l.) that cause changes in humidity and temperature along the gradient. The vegetation consists of a mosaic of forest fragments in different stages of ecological succession, with some cabrucas (cacao plantations where native woody plants are used for shading) and pastures. Preliminary floristic studies have identified 628 angiosperm species divided into 103 families (Amorim et al. 2009), and 173 species of ferns, as well as nine lycophytes; 44 of all species were new records for the State of Bahia and northeastern Brazil (Matos et al. 2010).

The climate in the region is the Af type of Köppen (1936) and is characterized as hot and humid without a dry season. During the study period, the average monthly rainfall was $160.9 \mathrm{~mm}(23.2-270.8 \mathrm{~mm})$, while the average minimum air temperature was $17.2^{\circ} \mathrm{C}\left(13.5-20.1{ }^{\circ} \mathrm{C}\right)$ and the maximum $25.8{ }^{\circ} \mathrm{C}\left(21.9-29.4^{\circ} \mathrm{C}\right)$. Summarized data is displayed in Figure 2. These data were collected using two rain gauges installed at the site, one in the lower area $(200 \mathrm{~m}$ a.s.l.) and another in the higher area $(850 \mathrm{~m}$ a.s.l.), plus eight minimum and maximum thermometers placed along the forest, four in the low area and four situated upland.

\section{Collection and analysis of data}

We conducted monthly field trips over six consecutive days from December 2009 to November 2010 and also recorded species during four other trips that lasted four days each, in July and December 2008, and February and May 2009, totaling 88 field days.

The inventory of amphibians was carried out using the following methods: (1) active visual and acoustic search (Heyer et al. 1994) via transects installed in the inner forest and streams; (2) pitfall traps (Cechin and Martins 2000); (3) active search in permanent and temporary ponds (Heyer et al. 1994); (4) opportunistic records done while the team moved along the trails, including specimens found by others. 


\section{Complex of RPPNs Serra Bonita}
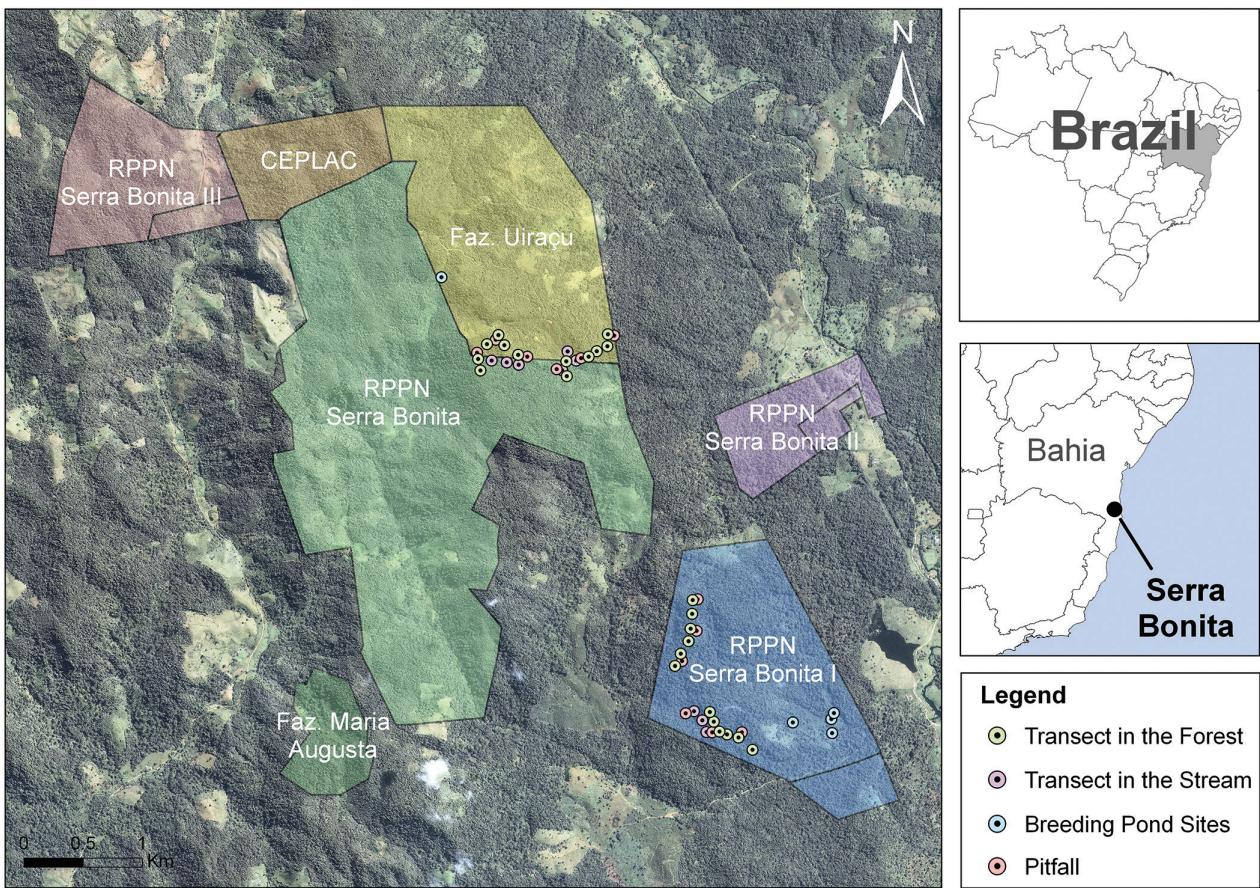

Legend
$\odot$ Transect in the Forest
$\odot$ Transect in the Stream
$\odot$ Breeding Pond Sites
$\odot$ Pitfall

Figure I. Map and sampling sites in the RPPN Serra Bonita, Bahia State, Northeastern Brazil.

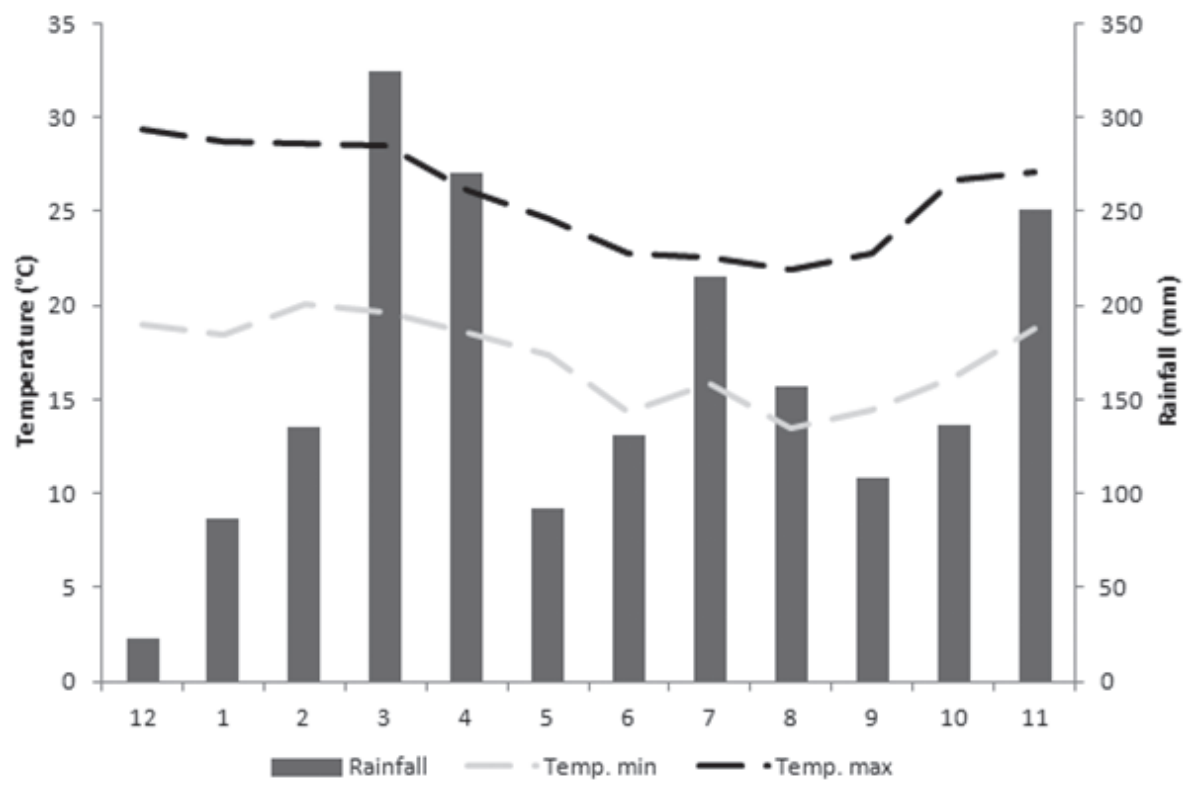

Figure 2. Rainfall data and minimum and maximum temperatures at the RPPN Serra Bonita between December 2009 and November 2010. 
From December 2009 to November 2010 we sampled 24 100-m transects placed in the inner forest and nine 50-m transects placed in streams. Active search was more frequently conducted by two researchers and occasionally by three. All transects were once inspected in every sampling month. Transects placed in the inner forest were traversed for 40 minutes, on average, totaling 192 hours $/ \mathrm{man}$. The transects placed in the streams, in turn, were traversed for 30 minutes, on average, totaling 54 hours $/ \mathrm{man}$.

We installed 12 pitfall traps, which were formed by four 100-L buckets shaped as "Y" and fitted with a one-meter-high canvas drift fence that connected them with five meter in length; the buckets remained open three nights per month over the year, totaling a sampling effort of 1728 buckets/day.

Five pools were occasionally sampled: two in the cabruca, another two in the pastures and another at the edge of the forest, with an overall sampling effort of about 25 hours. Temporary ponds formed on the used roads after heavy rain were also sampled.

The species that were found during reproductive activity were classified as having short (1-2 months), medium (3-7 months) and long (8-12 months) mating seasons. We considered the presence of males vocalizing as indicative of reproductive activity for species.

In order to verify a possible correlation between the monthly observed species richness and abiotic factors (rainfall, maximum and minimum temperatures), we used the Pearson's correlation coefficient. Normality was checked with the Shapiro-Wilk test. Correlations were computed using the SPSS 13.0 software. To calculate the number of species that could occur within the RPPN Serra Bonita, we used the shapes relative to the area of occurrence of amphibians provided by IUCN (2008) overlapped with the limits of the two municipalities in which the PRNP is located (Pau Brasil and Camacan), using the ArcGIS program.

All animals were collected under license provided by IBAMA and/or the Chico Mendes Institute for Biodiversity Conservation upon permission of the directors of local reserves. Vouchers were deposited at the Museu da Universidade Estadual de Santa Cruz (MZUESC), Ilhéus, Bahia, Brazil (Appendix I).

\section{Results}

Eighty amphibian species were found in the RPPN Serra Bonita: a single species of Gymnophiona (Siphonops annulatus - Siphonopidae) and 79 species of anurans, allocated into 14 families (Table 1; Figures 3-6). The species richness observed in the Serra Bonita mountain is the second highest recorded for the Atlantic Forest biome and almost doubles those found in other locations in the State of Bahia (Table 2). It is noteworthy that only the Ecological Reserve of Michelin had a similar sampling effort as the one applied to our study in the state of Bahia.

Among the species found in the study area that were already classified by the IUCN ( $\mathrm{n}=63)$, the vast majority $(\mathrm{n}=48 ; 76.1 \%)$ are considered as "Least Concern" and $20.6 \%(n=13)$ as data deficient to assess their conservation status 
Table I. Amphibian species found in the RPPN Serra Bonita, southern Bahia, Brazil. Caption. IUCN (International Union for Conservation of Nature and Natural Resources): DD = Deficient Data; LC = Least Concern; VU = Vulnerable; NT = Near Threatened. Habitat: LL = Leaf litter or understory; $S$ $=$ Streams $; \mathrm{P}=$ ponds $; \mathrm{B}=$ bromeliads or epiphytes; Mating Activity = Period of mating activity: $\mathrm{S}=$ short (1-2 months); $\mathrm{M}=$ medium (3-7 months); and L = long (8-12 months); Sampling Method: OE = Opportunistic encounters; TF $=$ Transect in the forest TS $=$ Transect in the streams; $\mathrm{P}=$ Pitfall; $\mathrm{BP}=$ Breeding pond sites. ${ }^{*}=$ species only found in the inner forests; $\dagger=$ only acoustic record; \# only recorded once or twice during the sampling.

\begin{tabular}{|c|c|c|c|c|}
\hline Family/Species & IUCN & Habitat & $\begin{array}{l}\text { Mating } \\
\text { Activity }\end{array}$ & $\begin{array}{l}\text { Sampling } \\
\text { Method }\end{array}$ \\
\hline \multicolumn{5}{|l|}{ AROMOBATIDAE } \\
\hline Allobates olfersioides (Lutz, 1925)* & VU & LLe $S$ & - & $\mathrm{P}, \mathrm{OE}$ \\
\hline \multicolumn{5}{|l|}{ BRACHYCEPHALIDAE } \\
\hline Brachycephalus pulex Napoli, Caramaschi, Cruz \& Dias, 2011* & - & LL & - & TF \\
\hline Ischnocnema verrucosa Reinhardt \& Lütken, $1862^{*}$ & DD & S, LL & S & TS, TF, P \\
\hline Ischnocnema sp. (gr. parva)* & - & LL & $S$ & TS, TF, P, OE \\
\hline \multicolumn{5}{|l|}{ BUFONIDAE } \\
\hline Rhinella crucifer (Wied-Neuwied, 1821) & LC & P, LL & M & TF, TS, P, OE, BP \\
\hline Rhinella granulosa (Spix, 1824) & LC & P, LL & $S$ & $\mathrm{OE}, \mathrm{BP}$ \\
\hline Rhinella hoogmoedi Caramaschi \& Pombal, 2006 & LC & P, S & $S$ & TF, TS, P, OE \\
\hline Rhinella jimi (Stevaux, 2002) & LC & LL & - & $\mathrm{OE}$ \\
\hline \multicolumn{5}{|l|}{ CENTROLENIDAE } \\
\hline Vitreorana eurygnatha (A. Lutz, 1925)* & LC & $S$ & $\mathrm{~L}$ & TS \\
\hline Vitreorana uranoscopa (Müller, 1924)* & LC & $S$ & $S$ & TS \\
\hline \multicolumn{5}{|l|}{ CERATOPHRYIDAE } \\
\hline Ceratophrys aurita (Raddi, 1823) \# & LC & LL & - & $\mathrm{OE}$ \\
\hline \multicolumn{5}{|l|}{ CRAUGASTORIDAE } \\
\hline Haddadus binotatus (Spix, 1824) & LC & LL & $\mathrm{L}$ & TF, TS, P, OE \\
\hline "Eleutherodactylus" bilineatus (Bokermann, 1975)* & LC & LL & $S$ & TF, $\mathrm{P}, \mathrm{OE}$ \\
\hline Pristimantis paulodutrai (Bokermann, 1975) & LC & LL & - & $\mathrm{OE}$ \\
\hline Pristimantis sp. $1^{*}$ & - & $\mathrm{B}$ & $\mathrm{L}$ & TF, TS, OE \\
\hline Pristimantis sp. $2^{*}$ & - & LL & $\mathrm{L}$ & TF, TS, OE \\
\hline Pristimantis sp. $3^{*} \#$ & - & LL & $S$ & $\mathrm{OE}$ \\
\hline Pristimantis vinhai (Bokermann, 1975) & LC & LL & $\mathrm{L}$ & TF, TS, OE \\
\hline \multicolumn{5}{|l|}{ ELEUTHERODACTYLIDAE } \\
\hline $\begin{array}{l}\text { Adelophryne mucronatus Lourenço-de-Moraes, Solé \& } \\
\text { Toledo, 2012* }\end{array}$ & - & LL & - & TF, TS, OE \\
\hline Adelophryne sp.* & - & LL & - & TF, TS, OE \\
\hline \multicolumn{5}{|l|}{ HEMIPHRACTIDAE } \\
\hline Gastrotheca sp.*\# & - & $\mathrm{B}$ & - & TF, OE \\
\hline Gastrotheca pulchra Caramaschi \& Rodrigues, 2007* & DD & $\mathrm{B}$ & - & TF, TS \\
\hline \multicolumn{5}{|l|}{ HYLIDAE } \\
\hline Aplastodiscus cf. weygoldti (Cruz \& Peixoto, 1985)* & NT & S & $\mathrm{L}$ & TF, TS, OE \\
\hline Aplastodiscus ibirapitanga (Cruz, Pimenta \& Silvano, 2003)* & LC & $S$ & M & TF, TS, OE, BP \\
\hline Bokermannohyla circumdata (Cope, 1871)* & LC & $S$ & $\mathrm{M}$ & TF, TS, OE \\
\hline Bokermannohyla lucianae (Napoli \& Pimenta, 2003)* & DD & $S$ & $\mathrm{~L}$ & TF, TS, OE \\
\hline Dendropsophus anceps (Lutz, 1929) & LC & $\mathrm{P}$ & $\mathrm{M}$ & TF, BP, OE \\
\hline Dendropsophus bipunctatus (Spix, 1824) \# & LC & $\mathrm{P}$ & S & $\mathrm{BP}$ \\
\hline Dendropsophus branneri (Cochran, 1948) & LC & $\mathrm{P}$ & $\mathrm{L}$ & $\mathrm{BP}$ \\
\hline
\end{tabular}




\section{Family/Species}

\begin{tabular}{|c|c|c|c|c|}
\hline & & & Activity & Method \\
\hline Dendropsophus elegans (Wied-Neuwied, 1824) & LC & $\mathrm{P}$ & $\mathrm{L}$ & BP, TS \\
\hline Dendropsophus giesleri (Mertens, 1950) & LC & $\mathrm{P}$ & $\mathrm{M}$ & $\mathrm{BP}$ \\
\hline Dendropsophus haddadi (Bastos \& Pombal, 1996) & LC & $\mathrm{P}$ & $\mathrm{L}$ & TS, BP \\
\hline Dendropsophus novaisi (Bokermann, 1968) \# & $\mathrm{DD}$ & $\mathrm{P}$ & $S$ & $\mathrm{BP}, \mathrm{TF}$ \\
\hline Dendropsophus minutus (Peters, 1872) & LC & $\mathrm{P}$ & S & $\mathrm{BP}$ \\
\hline Dendropsophus aff. oliveirai (Bokermann, 1963) & LC & $\mathrm{P}$ & $\mathrm{L}$ & $\mathrm{BP}$ \\
\hline Hypsiboas albomarginatus (Spix, 1824) & LC & $\mathrm{P}$ & $\mathrm{L}$ & $\mathrm{BP}$ \\
\hline Hypsiboas atlanticus (Caramaschi \& Velosa, 1996) $\dagger$ & LC & $\mathrm{P}$ & $\mathrm{M}$ & $\mathrm{BP}, \mathrm{OE}$ \\
\hline Hypsiboas crepitans (Wied-Neuwied, 1824) & LC & $\mathrm{P}$ & $\mathrm{M}$ & TF, BP \\
\hline Hypsiboas exastis (Caramaschi \& Rodrigues, 2003) \# & $\mathrm{DD}$ & $\mathrm{P}$ & S & $\mathrm{OE}$ \\
\hline Hypsiboas faber (Wied-Neuwied, 1821) & LC & $\mathrm{P}$ & M & TF, TS, BP, OE \\
\hline Hypsiboas pombali (Caramaschi, Pimenta \& Feio, 2004) & LC & $\mathrm{P}$ & $\mathrm{L}$ & TF, TS, OE, BP \\
\hline Hypsiboas semilineatus (Spix, 1824) & LC & $\mathrm{P}$ & S & $\mathrm{BP}$ \\
\hline Itapotihyla langsdorffii (Duméril \& Bibron, 1841) \# & LC & $\mathrm{P}$ & S & $\mathrm{BP}$ \\
\hline Phasmahyla spectabilis Cruz, Feio \& Nascimento, 2008* & $\mathrm{DD}$ & $S$ & $\mathrm{~L}$ & TF, TS \\
\hline Phyllodytes cf. maculosus Peixoto \& Cruz, $1988 \dagger$ & LC & B & $\mathrm{L}$ & TF, TS, OE \\
\hline $\begin{array}{l}\text { Phyllodytes melanomystax Caramaschi, Da Silva \& Britto- } \\
\text { Pereira, } 1992 \dagger\end{array}$ & LC & B & $\mathrm{L}$ & TF, TS, OE \\
\hline Phyllodytes wuchereri (Peters, 1873) & DD & $\mathrm{B}$ & $\mathrm{L}$ & TF, TS, OE \\
\hline Phyllodytes sp. 1 & - & B & $\mathrm{L}$ & TF, TS, OE \\
\hline Phyllodytes sp. $2 \dagger$ & - & $\mathrm{B}$ & $\mathrm{L}$ & TF, TS, OE \\
\hline Phyllodytes sp. $3 \dagger$ & - & $\mathrm{B}$ & $\mathrm{L}$ & TF, TS, OE \\
\hline Phyllodytes sp. $4 \dagger$ & - & B & $\mathrm{L}$ & TF, TS, OE \\
\hline Phyllomedusa burmeisteri Boulenger, 1882 & LC & $\mathrm{P}$ & $\mathrm{L}$ & TF, TS, OE \\
\hline Phyllomedusa nordestina Caramaschi, 2006 & DD & $\mathrm{P}$ & M & $\mathrm{BP}$ \\
\hline Phyllomedusa rohdei Mertens, 1926 & LC & $\mathrm{P}$ & M & $\mathrm{BP}$ \\
\hline Scinax argyreornatus (Miranda-Ribeiro, 1926) & LC & $\mathrm{P}$ & $S$ & $\mathrm{BP}, \mathrm{OE}$ \\
\hline Scinax juncae Nunes \& Pombal, 2010 & - & $\mathrm{P}$ & $\mathrm{M}$ & $\mathrm{BP}$ \\
\hline Scinax $x$-signatus (Spix, 1824) & LC & $\mathrm{P}$ & $\mathrm{S}$ & $\mathrm{BP}$ \\
\hline Scinax eurydice (Bokermann, 1968) & LC & $\mathrm{P}$ & - & TF, TS, BP, OE \\
\hline Scinax sp. $1 \#$ & - & $\mathrm{P}$ & S & $\mathrm{BP}$ \\
\hline Scinax sp. 2 (gr. rostratus) \# & - & - & - & $\mathrm{BP}$ \\
\hline Scinax strigilatus $($ Spix, 1824)* & $\mathrm{DD}$ & $S$ & S & TF, TS, OE \\
\hline Sphaenorhynchus prasinus Bokermann, 1973 \# & LC & $\mathrm{P}$ & $S$ & $\mathrm{BP}$ \\
\hline Trachycephalus mesophaeus (Hensel, 1867) & LC & $\mathrm{P}$ & $S$ & TF, BP, OE \\
\hline Trachycephalus nigromaculatus Tschudi, 1838* \# & LC & - & - & TF, OE \\
\hline \multicolumn{5}{|l|}{ HYLODIDAE } \\
\hline Crossodactylus sp.* & - & $S$ & - & TS, OE \\
\hline \multicolumn{5}{|l|}{ LEPTODACTYLIDAE } \\
\hline Adenomera cf. thomei Almeida \& Angulo, 2006* & LC & - & - & TS, P \\
\hline Leptodactylus cupreus Caramaschi, Feio \& São-Pedro, 2008 * \# & $\mathrm{DD}$ & $\mathrm{P}$ & - & $\mathrm{BP}$ \\
\hline Leptodactylus fuscus (Schneider, 1799) & LC & $\mathrm{P}$ & M & $\mathrm{BP}, \mathrm{OE}$ \\
\hline Leptodactylus cf. latrans (Steffen, 1815) & LC & $\mathrm{P}$ & $\mathrm{M}$ & TS, BP, OE, P \\
\hline Leptodactylus mystaceus (Spix, 1824) & LC & - & - & $\mathrm{OE}$ \\
\hline Physalaemus camacan Pimenta, Cruz \& Silvano, 2005 & $\mathrm{DD}$ & $\mathrm{P}$ & $\mathrm{M}$ & P, OE, BP \\
\hline Physalaemus erikae Cruz \& Pimenta, 2004 & LC & $\mathrm{P}$ & $\mathrm{M}$ & TF, P, OE, BP \\
\hline \multicolumn{5}{|l|}{ MICROHYLIDAE } \\
\hline Chiasmocleis crucis Caramaschi \& Pimenta, 2003 & DD & $\mathrm{P}$ & S & TF, P, BP \\
\hline
\end{tabular}




\begin{tabular}{l|c|c|c|c}
\hline Family/Species & IUCN & Habitat & $\begin{array}{c}\text { Mating } \\
\text { Activity }\end{array}$ & $\begin{array}{c}\text { Sampling } \\
\text { Method }\end{array}$ \\
\hline Stereocyclops histrio (Carvalho, 1954)*\# & DD & P & S & BP \\
\hline Stereocyclops incrassatus Cope, 1870 & LC & P & S & P, OE, BP \\
\hline ODONTOPHRYNIDAE & & & & \\
\hline Odontophrynus carvalhoi Savage \& Cei, 1965*\# & LC & S & S & OE \\
\hline Proceratophrys renalis (Miranda-Ribeiro, 1920)* & - & LL & - & OE \\
\hline Proceratophrys schirchi (Miranda-Ribeiro, 1937)* & LC & S, LL & L & TF, TS, P, OE \\
\hline PIPIDAE & & & & \\
\hline Pipa carvalhoi (Miranda-Ribeiro, 1937) \# & LC & P & - & BP \\
\hline SIPHONOPIDAE & & & & \\
\hline Siphonops annulatus (Mikan, 1820)* & LC & LL & - & TF, P, OE \\
\hline
\end{tabular}

Table 2. Number of anuran species found in areas of greatest richness within the Atlantic forest of Brazil and the state of Bahia (abbreviations for Brazilian states as follows: BA: Bahia; ES: Espírito Santo; SP: São Paulo; RJ: Rio de Janeiro). For studies providing the sampling effort in days and the total duration of the fieldwork we provided both.

\begin{tabular}{|c|c|c|c|c|}
\hline Localities & $\mathbf{N}$ & Time Sampled & $\begin{array}{c}\text { Area } \\
\left(\mathbf{k m}^{2}\right)\end{array}$ & References \\
\hline \multicolumn{5}{|l|}{ ATLANTIC RAIN FOREST } \\
\hline Municipality of Santa Teresa (ES) & 92 & $>10$ years & 711 & \begin{tabular}{|l} 
Almeida et al. 2011; \\
Rödder et al. 2007 \\
\end{tabular} \\
\hline RPPN Serra Bonita (BA) & 80 & 88 days (16 months) & 20 & This study \\
\hline Reserva Biológica de Paranapiacaba (SP) & 69 & * & 3.36 & Verdade et al. 2009 \\
\hline Municipality of Rio de Janeiro (RJ) & 68 & $>5$ years & 1356 & $\begin{array}{c}\text { Izecksohn and } \\
\text { Carvalho-e-Silva } \\
2001 \\
\end{array}$ \\
\hline Estaçăo Biológica da Boracéia (SP) & 67 & $>5$ years & 164.5 & Heyer et al. 1990 \\
\hline Parque Estadual Carlos Botelho (SP) & 65 & 76 days ( 1 year) & 377.9 & Forlani et al. 2010 \\
\hline Parque Estadual Turístico do Alto Ribeira (SP) & 60 & 15 days (3 months) & 357.1 & Araujo et al. 2010 \\
\hline \multicolumn{5}{|l|}{ STATE OF BAHIA } \\
\hline $\begin{array}{l}\text { Serra da Jibóia and Serra do Timbó - Elísio Medrado } \\
\text { and Amargosa }\end{array}$ & 53 & $\#$ & -100 & $\begin{array}{c}\text { Juncá 2006; } \\
\text { Camardelli and } \\
\text { Napoli } 2012\end{array}$ \\
\hline Reserva Ecológica da Michelin - Ituberá & 48 & 52 days (13 months) & 9.75 & $\begin{array}{l}\text { Camurugi et al. } \\
2010\end{array}$ \\
\hline $\begin{array}{l}\text { Parque Estadual da Serra do Conduru - Ilhéus, } \\
\text { Uruçuca and Itacaré }\end{array}$ & 45 & 13 days ( 2 months) & 92.7 & Pimenta 2005 \\
\hline Reserva Sapiranga - Mata de São João & $37-40$ & 36 days (8 months) & 6 & $\begin{array}{c}\text { Juncá 2006; } \\
\text { Bastazini et al. } 2007\end{array}$ \\
\hline RPPN Veracel - Porto Seguro & 39 & 4 days & 80.9 & $\begin{array}{l}\text { Silvano and Pimenta } \\
2003 \\
\end{array}$ \\
\hline Fazenda Vista Bela - Guaratinga & 34 & 4 days & 4.65 & \begin{tabular}{|l} 
Silvano and Pimenta \\
2003
\end{tabular} \\
\hline $\begin{array}{l}\text { Projeto de Assentamento Zumbi dos Palmares } \\
\text { - Camamu }\end{array}$ & 32 & 4 days & 18.3 & \begin{tabular}{|c|} 
Silvano and Pimenta \\
2003
\end{tabular} \\
\hline
\end{tabular}

* Mainly scientific data collections; \# richness estimated based on the literature and scientific data collections (Camardelli and Napoli 2012) and 12 days (4 months) of fieldworks (Juncá 2006). 


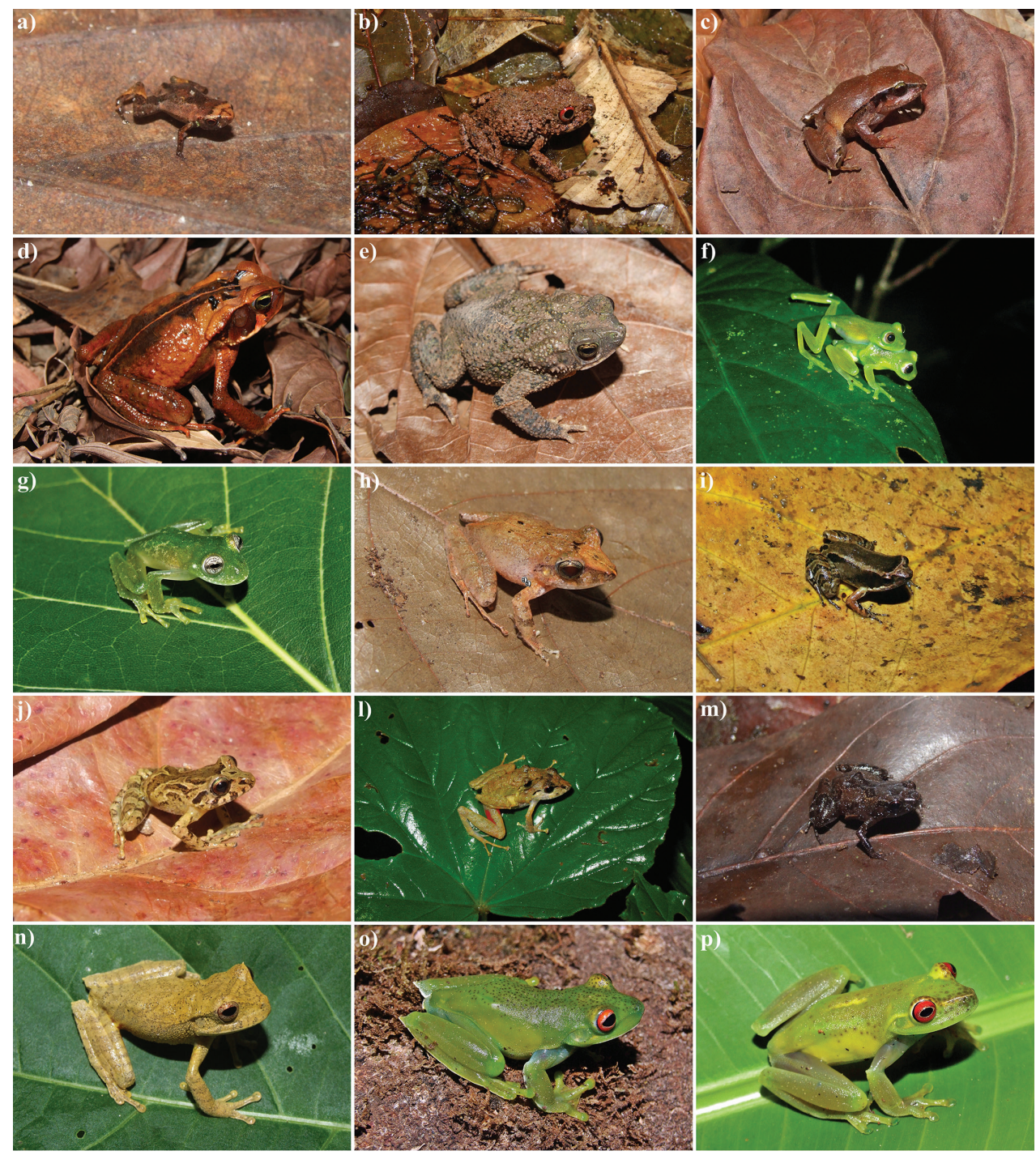

Figure 3. Anurans from the RPPN Serra Bonita, Bahia State, Northeastern Brazil. a Brachycephalus pulex b Ischnocnema verrucosa c Ischnocnema sp. 1 (gr. parva) d Rhinella crucifer e $R$. granulosa $\mathbf{f}$ Vitreorana eurygnatha $\mathbf{g} V$. uranoscopa $\mathbf{h}$ Haddadus binotatus $\mathbf{i}$ "Eleutherodactylus" bilineatus $\mathbf{j}$ Pristimantis sp. 1 I P. vinhai $\mathbf{m}$ Adelophryne sp. $\mathbf{n}$ Gastrotheca pulchra $\mathbf{o}$ Aplastodiscus ibirapitanga; and $\mathbf{p}$ A. cf. weygoldti. Photos by I. R. Dias.

(Table 1). Two species deserve special attention: Aplastodiscus cf. weygoldti and Allobates olfersioides, respectively, are currently considered near threatened and vulnerable to extinction by the IUCN (2013). A recent scientific assessment of the extinction risk of the Brazilian fauna concluded that Bokermannohyla lucianae and Phasmahyla spectabilis can be considered as near-threatened with extinction (Subirá et al. 2012). 

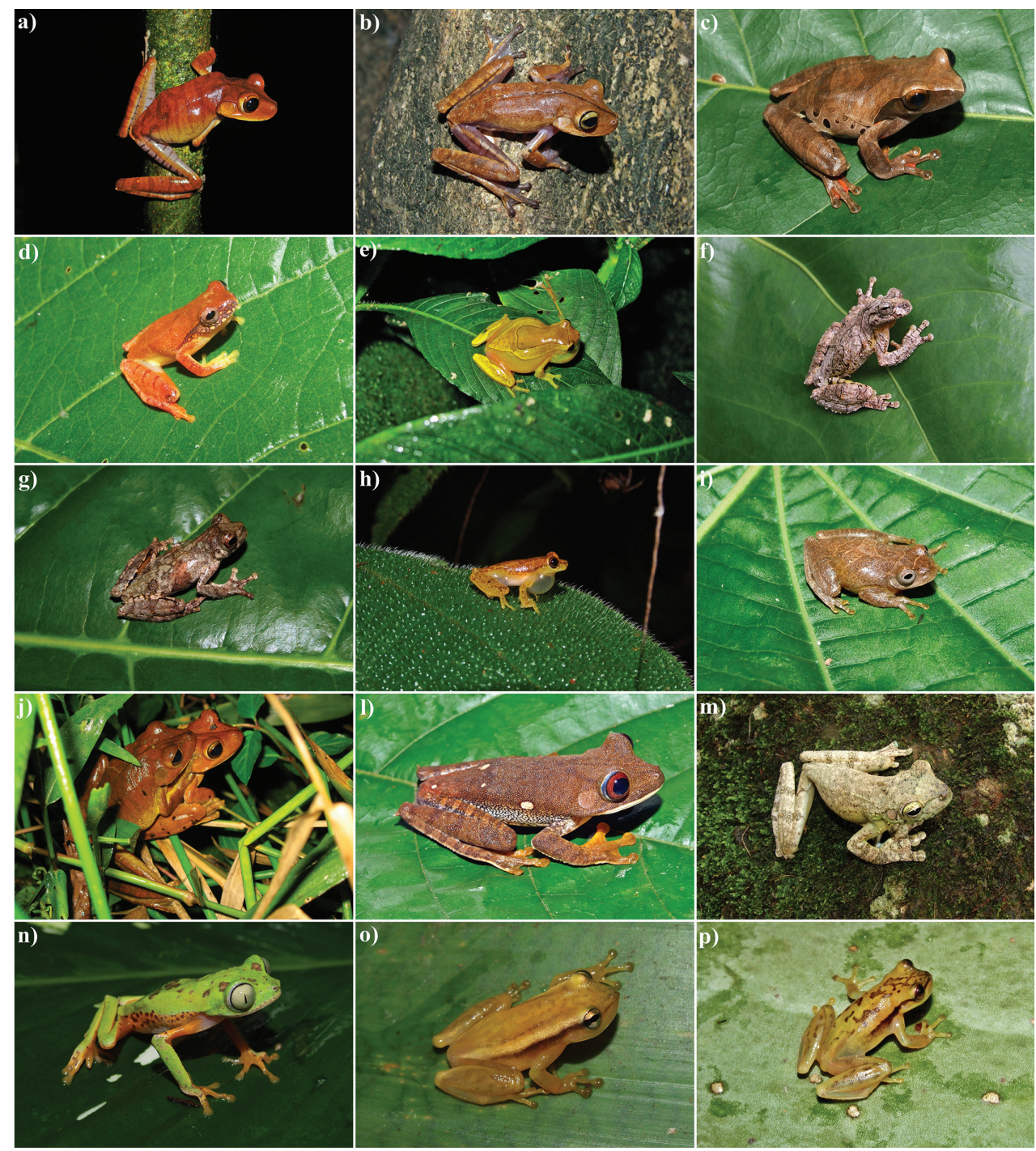

Figure 4. Anurans from the RPPN Serra Bonita, Bahia State, Northeastern Brazil. a Bokermannohyla circumdata $\mathbf{b}$ B. lucianae c Dendropsophus anceps $\mathbf{d} D$. bipunctatus $\mathbf{e} D$. elegans $\mathbf{f} D$. novaisi $\mathbf{g} D$. giesleri $\mathbf{h} D$. haddadi $\mathbf{i}$ D. minutus $\mathbf{j}$ Hypsiboas faber $\mathbf{I}$ H. semilineatus $\mathbf{m}$ Itapotihyla langsdorffii $\mathbf{n}$ Phasmahyla spectabilis o Phyllodytes wuchereri and p Phyllodytes sp. 1. Photos by I. R. Dias.

Amongst the species recorded during mating activity $(\mathrm{n}=61), 23$ exhibited prolonged mating $(37.7 \%)$ and the same number $(\mathrm{n}=23)$ was observed to mate on few occasions during the study period, whereas $24.5 \%$ showed an intermediate mating period (Table 1). Thirty-five of the species observed during mating activity were found in temporary or permanent ponds, twelve in streams, six in plant litter, and eight species were seen vocalizing in bromeliads and epiphytes (Table 1). 


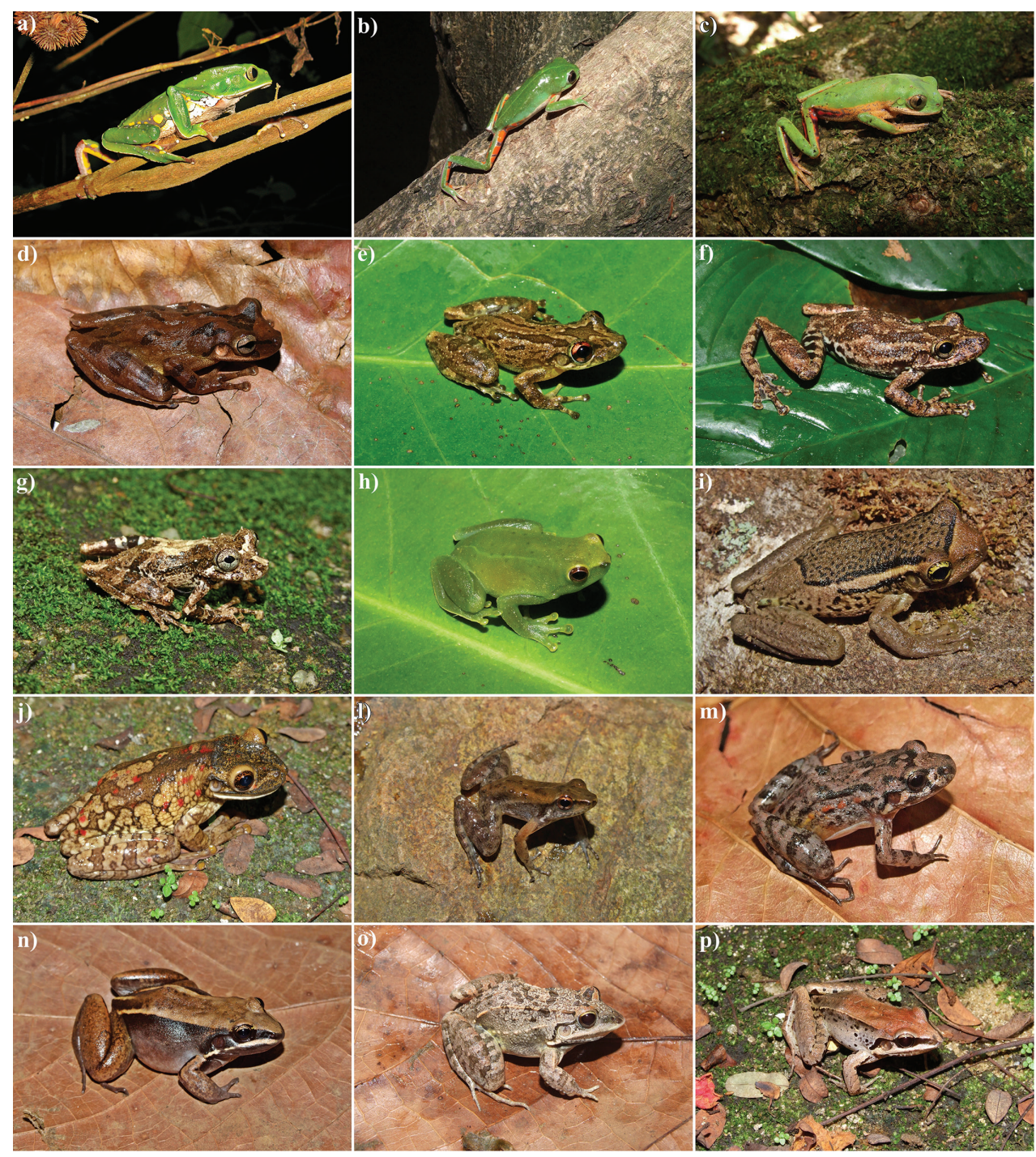

Figure 5. Anurans from the RPPN Serra Bonita, Bahia State, Northeastern Brazil. (a) Phyllomedusa burmeisteri $\mathbf{b} P$. nordestina $\mathbf{c} P$. rohdei $\mathbf{d}$ Scinax eurydice e Scinax sp. 1 f Scinax sp. 2 (gr. rostratus) g $S$. strigilatus $\mathbf{h}$ Sphaenorhynchus prasinus $\mathbf{i}$ Trachycephalus mesophaeus $\mathbf{j}$ T. nigromaculatus I Crossodactylus sp. $\mathbf{m}$ Adenomera cf. thomei $\mathbf{n}$ Leptodactylus cupreus $\mathbf{o}$ L. fuscus and $\mathbf{p}$ L. mystaceus. Photos by I. R. Dias.

The highest variety of species was recorded through opportunistic encounters (50 species). The search at breeding sites resulted in the registration of an intermediary of sampled species (38 species), however, with a high number of exclusive species (18 species), even using a smaller effort hour/man. Data on efficacy of the methodologies used can be checked in Table 3 .

A total of 16 species (20\%) was only recorded once or twice during the sampling (Table 1). Most of these species are explosive breeder and were sampled after heavy rainfall at the study area. 

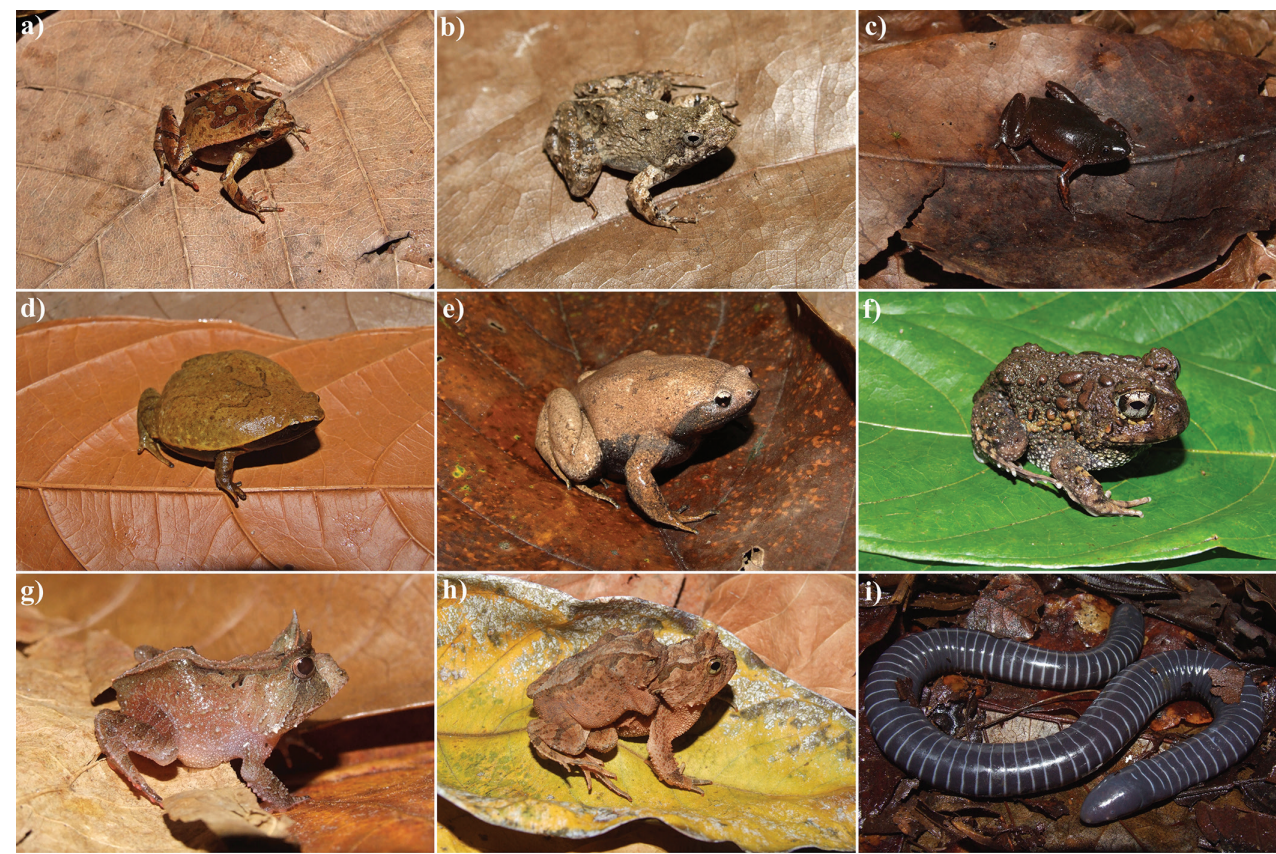

Figure 6. Anurans from the RPPN Serra Bonita, Bahia State, Northeastern Brazil. a Physalaemus camacan $\mathbf{b}$ P. erikae c Chiasmocleis crucis $\mathbf{d}$ Stereocyclops histrio e Stereocyclops incrassatus $\mathbf{f}$ Odontophrynus carvalhoi $\mathbf{g}$ Proceratophrys renalis $\mathbf{h}$ P. schirchi and i Siphonops annulatus. Photos by I. R. Dias.

Table 3. Number total and exclusive species sampled by each sampling method with the respective sampling effort.

\begin{tabular}{l|l|l|l}
\hline Sampling Method & Richness & Exclusive & Sampling Effort \\
\hline Opportunistic encounters & 50 & 7 & - \\
\hline Transect in the Forest & 40 & 1 & 192 (hours/man) \\
\hline Breeding pond sites & 38 & 18 & 25 (hours/man) \\
\hline Transect in the streams & 36 & 2 & 54 (hours/man) \\
\hline Pitfall & 15 & 0 & 1728 (buckets/day) \\
\hline
\end{tabular}

There was a significant positive correlation between richness and monthly rainfall ( $\mathrm{r}$ $=0.65, \mathrm{DF}=10, \mathrm{p}=0.027)$, but the same was not observed for the minimum $(\mathrm{r}=-0.22$, $\mathrm{DF}=10, \mathrm{p}=0.49)$ and the maximum $(\mathrm{r}=-0.31, \mathrm{DF}=10, \mathrm{p}=0.33)$ temperatures.

\section{Discussion}

Brazil harbors 946 amphibian species (Segalla et al. 2012) and near 543 of them have been recorded in the Atlantic Forest (Haddad et al. 2013). The State of Bahia is home to about 190 amphibian species. The amphibian community of RPPN Serra Bonita includes almost $15 \%$ of the overall species recorded in the Atlantic Forest and more than $40 \%$ of the species of Bahia State. Of the 20 anuran families that occur in Brazil (Frost 2014) only six 
(most of them Amazonian species) have not been sampled at the Serra Bonita mountain (Allophrynidae, Alsodidae, Ceuthomantidae, Cycloramphidae, Dendrobatidae and Ranidae). These are expressive numbers, since such high diversity was detected concentrated in the $20 \mathrm{~km}^{2}$ area forming the RPPN Serra Bonita.

The place known to show the largest amphibian richness in the Atlantic Forest is the municipality of Santa Teresa, Espírito Santo State, with 92 species (Rödder et al. 2007, Almeida et al. 2011). Yet, this amphibian diversity was obtained considering the entire municipality of Santa Teresa $\left(711 \mathrm{~km}^{2}\right)$, with a sampling effort of more than ten years, including records from different scientific collections. Thus, the amphibian diversity obtained in the RPPN Serra Bonita deserves special attention, as it comprises a smaller sampling area and effort. Even thus, the observed species richness is the second-highest ever recorded in a single study in the Atlantic Forest.

Most areas with high amphibian diversity in the Atlantic Forest are located in southeastern Brazil and are usually associated with mountainous locations (Heyer et al. 1990, Izecksohn and Carvalho-e-Silva 2001, Verdade et al. 2009, Forlani et al. 2010, Araujo et al. 2010). Aside from being the region where amphibians were better studied in Brazil, it concentrates the largest number of research groups working on amphibians (Rossa-Feres et al. 2011, Campos et al. in press).

Three out of the 80 species recorded at RPPN Serra Bonita (Eleutherodactylus " $b i$ lineatus", Pristimantis paulodutrai, P. vinhai) are endemic to the State of Bahia (Juncá and Pimenta 2004, Peixoto and Pimenta 2004a, Pimenta and Juncá 2004) and another six (Adelophryne mucronatus, Brachycephalus pulex, Chiasmocleis crucis, Physalaemus camacan, P. erikae, Stereocyclops histrio and Scinax strigilatus) are endemic to southern Bahia State (Cruz and Pimenta 2004, Pimenta et al. 2005b, Pimenta et al. 2007, Targino and Wild 2009, Napoli et al. 2011a, Lourenço-de-Moraes et al. 2012, Forlani et al. 2013). Phyllodytes wuchereri and Dendropsophus novaisi are species known from less than three localities (Caramaschi et al. 2004, Peixoto and Pimenta 2004b), whereas Bokermannohyla lucianae was only known from the municipality of Una (Dias et al. 2011). Thus, $15 \%$ of the species are endemic to the Bahia State and considering the species identified at specific level or as "cf.", $77.2 \%$ are endemic to the Atlantic Forest biome according to Haddad et al. (2013). Out of the 80 species recorded at the site, 29 were only recorded in the inner forests.

In this study we expand the geographic distribution of Vitreorana uranoscopa (Figure 3G) from Santa Teresa municipality, Espírito Santo State (Rödder et al. 2007) to the RPPN Serra Bonita (near $530 \mathrm{~km}$ north). Furthermore, two new species whose genera were only known to occur northward up to the Santa Teresa municipality were recently described from the State of Bahia. Crossodactylodes septentrionalis from Serra das Lontras (about $20 \mathrm{~km}$ from Serra Bonita in the municipality of Arataca) (Teixeira-Jr et al. 2013) and Brachycephalus pulex from RPPN Serra Bonita (Napoli et al. 2011a) both species being endemic to their type localities. To date, the two areas (municipality of Santa Teresa and RPPN Serra Bonita) have 33 species in common, but this number could even be larger. In addition, some species with northernmost 
distribution known to Santa Teresa have been found in the state of Bahia (Freitas et al. 2004, Orrico 2010, Camurugi et al. 2010).

Much of the shared amphibians between the two areas are composed of typical lowland species or species associated to mountainous regions but with a wide distribution range. The Santa Teresa region is located within the northern range of the Serra da Mantiqueira. The montane areas of southern Bahia do not belong to this mountain chain. The presence of restricted endemisms in both regions, especially species living at higher altitudes, may indicate that historic geomorphological and climatic factors may have contributed to turn these mountain ranges into key areas of speciation, as has been suggested for the northern range of the Serra da Mantiqueira (Napoli 2005, Napoli et al. 2011b). However, molecular studies with phylogeographic approaches are needed to allow a better understanding of the role of the bahian mountain ranges in the diversification of the local anuran fauna.

Amongst the 80 species found in the area, 18 were cited without a specific name or were classified as similar or described in groups of species (see Table 1). Some of these belong to groups that are taxonomically complex and difficult to identify (e.g. Adelophryne spp.; Crossodactylus spp.; Scinax spp.). The increase in research and scientific advances involving the specimens collected during this study can reveal the existence of new species or identify the presence of species to date unknown to exist in the State of Bahia. A recent analysis of samples collected during the development of this inventory resulted in the description of Brachycephalus pulex (Napoli et al. 2011a). Moreover, new species may be described for the region.

The sampling effort of our study was focused on transects installed within streams and inner forest fragments. Thus, some habitats and environments were not intensively sampled, such as the cabrucas, permanent and temporary ponds, as well as some areas of the RPPN complex that were not logistically feasible to be sampled. These areas had ponds and swamps in the inner forest, a feature that was not found in the areas sampled in our study. Future standardized effort including these environments might further enhance the richness of amphibians in the area.

In tropical regions, richness and mating activity are largely influenced by rainfall (Aichinger 1987, Duellman and Trueb 1994). Studies conducted in areas with seasonality detected a positive correlation between the sampled richness, rainfall and air temperature (Toledo et al. 2003, Santos et al. 2007, Kopp et al. 2010). Much of the amphibian community of Serra Bonita exhibited prolonged and intermediate mating activity (62\% of spp.). According to Crump (1974) this is the pattern expected for non-seasonal tropical regions. As the area does not present a marked seasonality, most species meet suitable conditions allowing them to mate during much of the year.

In this study we detected a positive correlation between the sampled species richness and the monthly rainfall. In addition, it is likely that there was an increase in the number of species sampled during the months with higher rainfall indexes due to the appearance of explosive breeding species (e.g. Dendropsophus novaisi, Stereocyclops incrassatus, Chiasmocleis crucis, Itapotihyla langsdorffi, Hypsiboas exastis, Scinax sp. 1 and Stereocyclops histrio). 


\section{Final remarks}

When comparing the amphibian species richness of RPPN Serra Bonita with that found at other sampled sites in the Bahia State, we notice a large disparity, since most locations exhibit less than half the number of species recorded in this study. This can be associated to the structural complexity of the sampled environment, which involves changes in temperature, rainfall, humidity and plant coverage along the altitudinal gradient in the study area, but also to the lack of systematic studies in most localities in the state of Bahia that still have large forest fragments and a mosaic of different ecosystems and biomes that need to be further explored from the scientific standpoint.

The largest Late Pleistocene refugium for amphibians has been estimated in southern Bahia and northern Espírito Santo states (Carnaval et al. 2009). During the quaternary glaciations, this zone of climatic stability probably allowed the coexistence and the diversification of a greater number of species in Serra Bonita, as compared to other places. Accordingly, the encounter of areas with a high concentration of amphibian species in this region was not unexpected. Serra Bonita may have been the first area to reveal such astonishing species richness, but other locations within the refugium have the same potential to support a high diversity of amphibians.

According to the IUCN (2008), the boundaries of the two municipalities of the studied RPPN (Camacan and Pau Brasil), are located within the area of geographical distribution of 26 other species (Aparasphenodon brunoi, Chiasmocleis carvalhoi, C. schubarti, Cycloramphus fuliginosus, Dendropsophus decipiens, D. nanus, Hypsiboas albopunctatus, Leptodactylus mystacinus, L. natalensis, L. spixi, L. viridis, Macrogenioglottus alipioi, Phyllodytes luteolus, Physalaemus crombiei, P. cuvieri, P. marmoratus, P. signifer, Pleurodema diplolister, Proceratophrys laticeps, Pseudopaludicola mystacalis, Scinax alter, S. fuscomarginatus, S. fuscovarius, Sphaenorhynchus palustris, S. pauloalvini and Thoropa miliaris). These species have not been found during our field activities, but may potentially occur in the region. Most of them are typically encountered in lowland areas. Despite being a mountain with well preserved forests on its top several smaller forest fragments and abandoned cacao plantations still remain in the lowland areas of the RPPN, offering potential habitat for theses species. Two recently described species (Crossodactylodes septentrionalis and Dendrophryniscus oreites) from Serra das Lontras (ca. $20 \mathrm{~km}$ from Serra Bonita) and the species Gastrotheca recava and G. megacephala (Teixeira-Jr et al. 2012, Teixeira-Jr et al. 2013, Recoder et al. 2010) possibly also may occur in the study area. Thus, we estimate that Serra Bonita may be the home for 100-110 amphibian species, representing one of the largest diversities for the group in the world.

The number of research institutes with graduate programs and professionals working on biodiversity in the State of Bahia has steadily increased during the last decade. The amount of information is expected to significantly increase in the coming years. Nonetheless, if we consider the vast extent of the territorial borders of Bahia, this increase in physical and human resources should be encouraged and further increased in order that we can better understand the diversity of anuran fauna in the State of Bahia. 
A further step is to encourage funding agencies to sponsor scientific expeditions and medium and long term studies in the State - mostly basic studies - such as inventories of the local fauna.

Serra Bonita has a total area of 7500 hectares. One goal of the Uiraçu Institute is to protect about half that area. To date, about 2000 ha are under protection of the institute, which manages and protects the areas under a free lease agreement. Coupled with the wide diversity and endemism of amphibians found in the area, the finding of new species and new records for Bahia State make Serra Bonita a hotpoint for amphibians within the Atlantic Forest hotspot and, consequently, a place for the implementation of priority conservation measures aiming the increase of the protected area.

\section{Acknowledgements}

We are thankful to Vitor Becker and Clemira Souza for their help during fieldwork and for opening the doors of RPPN Serra Bonita, as well as all the staff of the Uiraçu Institute, in particular the park rangers Marivaldo Mota, Gerson Santos, Ednilson Barreto and Ronoaldo Araújo, as well as the reserve employees Ronison Rodrigues and Marcos Cardoso for their excellent aid during fieldworks. In the same way, we are thankful to the colleagues Amanda Lantyer-Silva, Caio Mendes, Danilo Ruas, Raoni Rebouças, Euvaldo Marciano-Junior, Edirlan Santos, Filipe Gudinho and Vinicius Lima for the discussions and collaboration during field activities. We are also thankful to Bianca Berneck, Bruno Pimenta, Célio Haddad, Clarissa Canedo, Diego Santana, Ivan Nunes, José Pombal, Marcelo Napoli, Mauricio Forlani, Miguel Rodrigues, Ricardo Lourenço-de-Moraes, Ulisses Caramaschi and Victor Dill for collaborating in the identification of some species. We further thank Andrés Egea Serrano, Dennis Rödder and Marcelo Felgueiras Napoli for their valuable comments on the manuscript. This work was part of the project "Characterization of terrestrial vertebrates at the RPPN Complex of Serra Bonita as an aid for its effective management" supported by the Boticário Group Foundation for Nature Protection (Project No. 0818_20091). IRD is grateful to CAPES and FAPESB for fellowships.

\section{References}

Aichinger M (1987) Annual activity patterns of anurans in a seasonal neotropical environment. Oecologia 71(4): 583-592. doi: 10.1007/BF00379302

Almeida AP, Gasparini JL, Peloso PLV (2011) Frogs of the state of Espírito Santo, southeastern Brazil - The need for looking at the 'coldspots'. Check List 7(4): 542-560. http://www. checklist.org.br/getpdf?SL021-11

Amorim AMA, Fiaschi P, Jardim JG, Thomas WW, Clifton B, Carvalho AMV (2005) The Vascular Plants of a Forest Fragment in Southern Bahia, Brazil. SIDA Contributions to Botany 21(3): 1726-1752. 
Amorim AMA, Jardim JG, Lopes MMM, Fiaschi P, Borges RAX, Perdiz RO, Thomas WW (2009) Angiospermas em remanescentes de floresta montana no sul da Bahia, Brasil. Biota Neotropica 9: 313-348. doi: 10.1590/S1676-06032009000300028

Amorim AMA, Leme E (2009) Two new species of Quesnelia (Bromeliaceae: Bromelioideae) from the Atlantic Rain Forest of Bahia, Brazil. Brittonia 61(1): 14-21. doi: 10.1007/ s12228-008-9049-5

Araujo CO, Condez TH, Bovo RP, Centeno FC, Luiz AM (2010) Amphibians and reptiles of the Parque Estadual Turístico do Alto Ribeira (PETAR), SP: an Atlantic Forest remnant of Southeastern Brazil. Biota Neotropica 10(4): 257-274. doi: 10.1590/S167606032010000400031

Bastazini CV, Munduruca JFV, Rocha PLB, Napoli MF (2007) Which environmental variables better explain changes in anuran community composition? a case study in the restinga of Mata de São João, Bahia, Brazil. Herpetologica 63: 459-471. doi: 10.1655/0018-0831(2007)63[459:WEVBEC]2.0.CO;2

Becker CG, Fonseca CR, Haddad CFB, Batista RF, Prado PI (2007) Habitat Split and the Global Decline of Amphibians. Science 318: 1775-1777. doi: 10.1126/science.1149374

Bencke GA, Mauricio GN, Develey PR, Goerck JM (2006) Áreas importantes para a conservação das aves no Brasil: Parte I-estados do domínio da Mata Atlântica, incluindo áreas de Cerrado, Caatinga e Pampas. SAVE Brasil, São Paulo, 494 pp.

Blaustein AR, Johnson PTJ (2003) The complexity of deformed amphibians. Frontiers in Ecology and the Environment 2: 87-94. doi: 10.1890/1540-9295(2003)001[0087:TC ODA]2.0.CO;2

Brown KS (1991) Conservation of neotropical environments: insects as indicators. In: Collins NM, Thomas JA (Eds) Conservation of Insects and Their Natural Environments, R.E.S. Symposium Nr. 15. Academic Press, London, UK, 350-404.

Campos FS, Brito D, Solé M (2013) Threatened Amphibians and Their Conservation Status within the Protected Area Network in Northeastern Brazil. Journal of Herpetology 47: 277-285. doi: 10.1670/11-158

Campos FS, Brito D, Solé M (in press) Diversity patterns, research trends and mismatches of the investigative efforts to amphibian conservation in Brazil. Anais da Academia Brasileira de Ciências 86(4).

Camurugi F, Lima TM, Mercês EA, Juncá FA (2010) Anuros da Reserva Ecológica da Michelin, Município de Igrapiúna, Estado da Bahia, Brasil. Biota Neotropica 10(2): 305-312. doi: 10.1590/S1676-06032010000200032

Caramaschi U, Peixoto O, Rodrigues MT (2004) Revalidation and redescription of Phyllodytes wuchereri (Peters, 1873) (Amphibia, Anura, Hylidae). Arquivos do Museu Nacional, Rio de Janeiro 62(2): 185-191.

Caramaschi U, Orrico VGD, Faivovich J, Dias IR, Solé M (2013) A new species of Allophryne (Anura: Allophrynidae) from the Atlantic Rain Forest Biome of eastern Brazil. Herpetologica 69: 480-491. doi: 10.1655/HERPETOLOGICA-D-13-00029

Carnaval AC, Hickerson MJ, Haddad CFB, Rodrigues MT, Moritz C (2009) Stability predicts genetic diversity in the Brazilian Atlantic Forest hotspot. Science 323(5915): 785-789. doi: $10.1126 /$ science. 1166955 
Cechin SZ, Martins M (2000) Eficiência de armadilhas de queda (pitfall traps) em amostragem de anfíbios e répteis no Brasil. Revista Brasileira de Zoologia 17: 729-740. doi: 10.1590/ S0101-81752000000300017

Crump ML (1974) Reproductive strategies in a tropical anuran community. Miscellaneous publication - University of Kansas, Museum of Natural History 61: 1-68.

Cruz CAG, Feio RN (2007) Endemismos em anfíbios em áreas de altitude na Mata Atlântica no sudeste do Brasil. In: Nascimento LB, Oliveira ME (Eds) Herpetologia no Brasil II. 1 ed. Belo Horizonte: Sociedade Brasileira de Herpetologia 1: 117-126.

Cruz CAG, Pimenta BVS (2004) A new species of Physalaemus Fitzinger, 1826 from Southern Bahia state, Brazil (Anura, Leptodactylidae). Journal of Herpetology 38(4): 480-486. doi: 10.1670/214-02A

Dias IR, Medeiros TT, Solé M, Pimenta BVS (2011) Amphibia, Anura, Hylidae, Bokermannohyla lucianae (Napoli and Pimenta 2003): Distribution extension and geographic distribution map. Check List 7(2): 108-109. http://www.checklist.org.br/getpdf?NGD115-10

Dias IR, Vilaça TRA, Silva JRS, Barbosa RS, Solé M (2010) Amphibia, Anura, Hylidae, Trachycephalus nigromaculatus Tschudi, 1838: Distribution extension. Check List 6(3): 412-413. http://checklist.org.br/getpdf?NGD013-10

Droege S, Cyr A, Larivée J (1998) Checklists: an under-used tool for the inventory and monitoring of plants and animals. Conservation Biology 12(5): 1134-1138. doi: 10.1046/j.15231739.1998.96402.x

Duellman WE, Trueb L (1994) Biology of Amphibians. The Johns Hopkins University Press, Baltimore, Maryland, USA, 670 pp.

Forlani MC, Bernardo PH, Haddad CBF, Zaher H (2010) Herpetofauna of the Carlos Botelho State Park, São Paulo State, Brazil. Biota Neotropica 10(3): 265-309. doi: 10.1590/ S1676-06032010000300028

Forlani MC, Mendes CVM, Dias IR, Ruas DS, Tonini JFR, de Sá RO (2013) The advertisement calls and distribution of two sympatric species of Chiasmocleis (Méhely 1904) (Anura, Microhylidae, Gastrophryninae) from the Atlantic Forest. South American Journal of Herpetology 8(1): 46-51. doi: 10.2994/SAJH-D-12-00027.1

Freitas MA, Silva TFS, Argolo AJS (2004) Geographic Distribution: Hyalinobatrachium eurygnathum (Rio Glass Frog). Herpetology Review 35(3): 281.

Frost DR (2014) Amphibian Species of the World: an Online Reference. Version 6.0. Electronic Database accessible at http://research.amnh.org/herpetology/amphibia/index.html [acessed on 01 March 2014] American Museum of Natural History, New York, USA.

Garda AA, Costa TB, Santos-Silva CR, Mesquita DO, Faria RG, Conceição BM, Silva IRS (2013) Herpetofauna of protected areas in the Caatinga I: Raso da Catarina Ecological Station (Bahia, Brazil). Check List 9(2): 405-414. http://www.checklist.org.br/getpdf?SL115-12

Haddad CFB (1998) Biodiversidade dos Anfíbios no Estado de São Paulo. In: Joly CA, Bicudo CEM (Eds) Biodiversidade do Estado de São Paulo, Brasil: síntese do conhecimento ao final do século XX. FAPESP, São Paulo, 17-26.

Haddad CFB, Toledo LF, Prado CPA, Loebmann D, Gasparini JL, Sazima I (2013) Guide to the Amphibians of the Atlantic Forest: Diversity and Biology. Anolisbooks, São Paulo, 544 pp. 
Haffer J (1974) Avian speciation in tropical South America. Publication of the Nuttall Ornithological Club 14: 1-390. http://www.jstor.org/stable/4160667

Heyer WR, Rand AS, Cruz CAG, Peixoto OL, Nelson CE (1990) Frogs of Boracéia. Arquivos de Zoologia 31: 231-410.

Heyer WR, Donelly MA, Mcdiarmid RW, Hayek LAC, Foster MS (1994) Measuring and monitoring biological diversity: Standart methods for Amphibians. Smithsonian Institution Press, Washington.

Hoffmann M, Hilton-Taylor C, Angulo A et al. (2010) The impact of conservation on the status of the world's vertebrates. Science 330(6010): 1503-1509. doi: 10.1126/science.1194442

Instituto Uiraçu (2009) Serra Bonita http://www.uiracu.org.br/serrabonita.html [acessed on 01 March 2014]

IUCN (2008) An Analysis of Amphibians on the 2008 IUCN Red List. www.iucnredlist.org/ amphibians

IUCN (2013) IUCN Red List of Threatened Species. Version 2013.2. www.iucnredlist.org [acessed on 01 March 2014]

Izecksohn E, Carvalho-e-Silva SP (2010) Anfíbios do município do Rio de Janeiro. 2nd ed. Rio de Janeiro (RJ).

Juncá FA (2005) Anfíbios e Répteis. In: Juncá FA, Funch L, Rocha W (Eds) Biodiversidade e Conservação da Chapada Diamantina. Série Biodiversidade. 1 ed. Ministério do Meio Ambiente, Distrito Federal-Brasília, 13: 339-376.

Juncá FA (2006) Diversidade e uso de hábitat por anfíbios anuros em duas localidades de Mata Atlântica, no norte do Estado da Bahia. Biota Neotropica 6(2): 1-8. doi: 10.1590/S167606032006000200018

Juncá FA, Pimenta B (2004) Ischnocnema bilineata. In: IUCN 2013. IUCN Red List of Threatened Species. Version 2013.2. http://www.iucnredlist.org [acessed on 01 March 2014]

Kats LB, Ferrer RP (2003) Alien predators and amphibian declines: review of two decades of science and transition to conservation. Diversity and Distributions 9: 99-110. doi: 10.1046/j.1472-4642.2003.00013.x

Kopp K, Signorelli L, Bastos RP (2010) Distribuição temporal e diversidade de modos reprodutivos de anfíbios anuros no Parque Nacional das Emas e entorno, estado de Goiás, Brasil. Iheringia 100: 192-200. doi: 10.1590/S0073-47212010000300002

Köppen WP (1936) Das geographische System der Klimate. Gebrüder Borntraeger, Berlin, 44 pp. Lantyer-Silva ASF, Siqueir-Jr S, Zina J (2013) Checklist of amphibians in a transitional area between the Caatinga and the Atlantic Forest, central-southern Bahia, Brazil. Check List 9 (4): 725-732. http://www.checklist.org.br/getpdf?SL099-12

Lemes P, Melo AS, Loyola RD (2014) Climate change threatens Protected Areas of the Atlantic Forest. Biodiversity and Conservation 23: 357-368. doi: 10.1007/s10531-013-0605-2 Lips KR, Green DE, Papendick R (2003) Chytridiomycosis in wild frogs from southern Costa Rica. Journal of Herpetology 37: 215-218. doi: 10.1670/0022-1511(2003)037[0215:CI WFFS]2.0.CO;2

Lourenço-De-Moraes R, Solé M, Toledo LF (2012) A new species of Adelophryne Hoogmoed and Lescure 1984 (Amphibia: Anura: Eleutherodactylidae) from southern Bahia, Brazil. Zootaxa 3441: 59-68. 
Loyola RD, Kubota U, Lewinsohn TM (2007) Endemic vertebrates are the most effective surrogate for identifying conservation priorities among Brazilian ecoregions. Diversity and Distributions 13: 389-396. doi: 10.1111/j.1472-4642.2007.00345.x

Machado AFP, Vianna-Filho MD, Amorim AMA, Romaniuc-Neto S (2013) A new species Sorocea (Moraceae), from Montane rainforest at Bahia state, Brazil. Systematic Botany 58: 01-05.

Martinelli G (2007) Mountain biodiversity in Brazil. Revista Brasileira de Botânica 30: 587-597. doi: 10.1590/S0100-84042007000400005

Martini AMZ, Fiaschi P, Amorim AMA, Paixão JL (2007) A hot-point within a hot-spot: a high diversity site in Brazil's Atlantic forest. Biodiversity and Conservation 16: 31113128. doi: 10.1007/s10531-007-9166-6

Matos FB, Amorim AMA, Labiak PH (2010) The Ferns and Lycophytes of a Montane Tropical Forest in Southern Bahia, Brazil. Journal of the Botanical Research Institute of Texas 4: 335-348.

Morais AR, Siqueira MN, Lemes P, Maciel MM, Marco P, Brito D (2013) Unraveling the conservation status of Data Deficient species. Biological Conservation 166: 98-102. doi: 10.1016/j.biocon.2013.06.010

Murray-Smith C, Brummitt NA, Oliveira-Filho AT, Bachman S, Moat J, Lughadha EMN, Lucas EJ (2008) Plant diversity hotspots in the Atlantic Coastal Forests of Brazil. Conservation Biology 23: 151-163. doi: 10.1111/j.1523-1739.2008.01075.x

Myers N, Mittermeyer RA, Fonseca GAB, Kent J (2000) Biodiversity hotspots for conservation priorities. Nature 403: 853-858. doi: 10.1038/35002501

Napoli M, Encarnação L, Cunha M, Abreu R, Herrera J (2011b) Paradoxical geographic distributions, new record, and corrections of Bokermannohyla circumdata (Cope, 1870) and $B$. caramaschii (Napoli, 2005) (Amphibia: Anura: Hylidae). Herpetology notes 4: 105-109. http://www.herpetologynotes.seh-herpetology.org/Volume4_PDFs/Napoli_Herpetology_Notes_Volume4_pages105-109.pdf

Napoli MF, Caramaschi U, Cruz CAG, Dias IR (2011a) A new species of flea-toad, genus Brachycephalus Fitzinger (Amphibia: Anura: Brachycephalidae), from the Atlantic rainforest of southern Bahia, Brazil. Zootaxa 2739: 33-40.

Napoli MF (2005) A new species allied to Hyla circumdata (Anura: Hylidae) from Serra da Mantiqueira, Southeastern Brazil. Herpetologica 61(1): 63-69. doi: 10.1655/03-41

Orrico VD (2010) Amphibia, Anura, Brachycephalidae, Ischnocnema verrucosa Reinhardt and and Lütken, 1862: Distribution extension to northeastern Brazil. Check List 6: 246-247. http://www.checklist.org.br/getpdf?NGD120-09

Peixoto O, Pimenta B (2004a) Ischnocnema vinhai. IUCN Red List of Threatened Species. Version 2013.2. http://www.iucnredlist.org [acessed on 01 March 2014]

Peixoto LO, Pimenta B (2004b) Dendropsophus novaisi. IUCN Red List of Threatened Species. Version 2013.2. http://www.iucnredlist.org [acessed on 01 March 2014]

Pimenta B, Juncá FA (2004) Ischnocnema paulodutrai. IUCN Red List of Threatened Species. Version 2013.2. http://www.iucnredlist.org [acessed on 01 March 2014]

Pimenta BVS, Haddad CFB, Nascimento LB, Cruz CAG, Pombal-Jr JP (2005a) Comment on status and trends of amphibian declines and extinctions worldwide. Science 309: 1999. doi: $10.1126 /$ science. 1112996 
Pimenta BVS, Cruz CAG, Silvano DL (2005b) A new species of the genus Physalaemus Fitzinger, 1826 (Anura, Leptodactylidae) from the Atlantic Rain Forest of southern Bahia, Brazil. Amphibia-Reptilia 26(2): 201-210. doi: 10.1163/1568538054253483

Pimenta B (2005c) Anfíbios. In: Schiavetti A, Leopoldino F, Paglia A, Pinto LP (Eds) Plano de Manejo do Parque Estadual da Serra do Conduru. BAHIA/ SEMARH - Secretária de Meio Ambiente e Recursos Hídricos, 320 pp.

Pimenta BVS, Faivovich J, Pombal-Jr JP (2007) On the identity of Hyla strigilata Spix, 1824 (Anura: Hylidae): redescription and holotype designation for a 'ghost' taxon. Zootaxa 1441: 35-49.

Pounds JA, Bustamante MR, Coloma LA, Consuegra JA, Fogden MPL, Foster PN, La Marca E, Masters KL, Merino-Viteri A, Puschendorf R, Ron SR, Sánchez-Azofeifa GA, Still CJ, Young BE (2006) Widespread amphibian extinctions from epidemic disease driven by global warming. Nature 439: 161-167. doi: 10.1038/nature04246

Protázio AS, Santos-Protázio A, Silva CRS, Ribeiro ES, Nogueira SEM, Moura GJB (2009) Anurofauna do Município de Paulo Afonso - BA, Bioma Caatinga, Nordeste do Brasil. Revista Nordestina de Zoologia 4(2): 31-38. http://www.revistanordestinadezoologia. com/images/stories/vertebrados/Artigo_04.pdf

Recoder RS, Teixeira-Jr M, Cassimiro J, Camacho AG, Rodrigues MT (2010) A new species of Dendrophryniscus (Amphibia, Anura, Bufonidae) from the Atlantic Rainforest of southern Bahia, Brazil. Zootaxa 2642: 36-44

Ribeiro MC, Metzger JP, Martensen AC, Ponzoni FJ, Hirota MM (2009) The Brazilian Atlantic Forest: How much is left, and how is the remaining forest distributed? Implications for conservation. Biological Conservation 142: 1141-1153. doi: 10.1016/j.biocon.2009.02.021

Rocha DSB, Amorim AMA (2012) Heterogeneidade altitudinal na Floresta Atlântica setentrional: um estudo de caso no sul da Bahia, Brasil. Acta Botanica Brasílica 26: 309-327.

Rödder D, Teixeira RL, Ferreira RB, Dantas RB, Pertel W, Guarneire GJ (2007) Anuran hotspots: the municipality of Santa Teresa, Espírito Santo, southeastern Brazil. Salamandra 43 (2): 91-110.

Rossa-Feres DC, Sawaya RJ, Faivovich J, Giovanelli JGR, Brasileiro CA, Schiesari L, Alexandrino J, Haddad CFB (2011) Anfíbios do Estado de São Paulo, Brasil: conhecimento atual e perspectivas. Biota Neotropica 11(1a): 1-19. doi: 10.1590/S1676-06032011000500004

Santos TG, Rossa-Feres DC, Casatti L (2007) Diversidade e distribuição espaço-temporal de anuros em região com pronunciada estaçáo seca do sudeste do Brasil. Iheringia 97(1): 37-49. doi: 10.1590/S0073-47212007000100007

Segalla MV, Caramaschi U, Cruz CAG, Garcia PCA, Grant T, Haddad CFB, Langone J (2012) Brazilian amphibians - List of species. Sociedade Brasileira de Herpetologia. http:// www.sbherpetologia.org.br/?page_id=644 [accessed on 10 May 2014]

Silvano DL, Pimenta BVS (2003) Diversidade de anfíbios na Mata Atlântica do Sul da Bahia. In: Prado PI, Landau EC, Moura RT, Pinto LPS, Fonseca GAB, Alger K (Eds) Corredor de Biodiversidade na Mata Atlântica do Sul da Bahia CD-ROM, Ilhéus, IESB/CI/CABS/ UFMG/UNICAMP. 
Stuart S, Chanson JS, Cox NA, Young BE, Rodrigues ASL, Fishman DL, Waller RW (2004) Status and trends of amphibian declines and extinctions worldwide. Science 306: 17831786. doi: $10.1126 /$ science. 1103538

Subirá RJ, Souza ECF, Guidorizzi CE, Almeida MP, Almeida JB, Martins DS (2012) Avaliação Científica do Risco de Extinção da Fauna Brasileira - Resultados Alcançados em 2012. Biodiversidade Brasileira: Edição mista: manejo de recursos vegetais e avaliação do estado de conservação de espécies 2(2): 17-24.

Targino M, Wild E (2009) Amphibia, Anura, Microhylidae, Hyophryne histrio: Distribution extension. Check List 5(1): 24-26. www.checklist.org.br/getpdf?NGD136-08

Teixeira-Jr M, Vechio FD, Recoder RS, Carnaval ACOQ, Strangas M, Damasceno RP, Sena MA, Rodrigues MT (2012) Two new species of marsupial tree-frogs genus Gastrotheca Fitzinger, 1843 (Anura, Hemiphractidae) from the Brazilian Atlantic Forest. Zootaxa 3437: 1-23.

Teixeira-Jr M, Recoder RS, Amaro RC, Damasceno RP, Cassimiro J, Rodrigues MT (2013) A new Crossodactylodes Cochran, 1938 (Anura: Leptodactylidae: Paratelmatobiinae) from the highlands of the Atlantic Forests of southern Bahia, Brazil. Zootaxa 3702(5): 459-472. doi: 10.11646/zootaxa.3702.5.5

Thomas WW, Carvalho AMV, Amorim AMA, Garrison J, Albeláez AL (1998) Plant endemism in two forests in southern Bahia, Brazil. Biodiversity and Conservation 7: 311-322. doi: 10.1023/A:1008825627656

Thomas WW, Carvalho AMV, Amorim AMA, Hanks JG, Santos TS (2008) Diversity of woody plants in the Atlantic coastal forest of southern Bahia, Brazil. Memoirs of the New York Botanical Garden 100: 21-66.

Toledo LF, Zina J, Haddad CFB (2003) Distribuição espacial e temporal de uma comunidade de anfíbios anuros do município de Rio Claro, São Paulo, Brasil. Holos Environment 3 (2): 136-149.

Trindade-Filho J, Carvalho RA, Brito D, Loyola RD (2012) How does the inclusión of Data Deficient species change conservation priorities for amphibians in the Atlantic Forest? Biodiversity and Conservation 21: 2709-2718. doi: 10.1007/s10531-012-0326-y

Valdujo PH, Recoder RS, Vasconcellos MM, Portella AS (2009) Amphibia, Anura, Sao Desidério, western Bahia uplands, northeastern Brazil. Check List 5(4): 903-911. http://www. checklist.org.br/getpdf?SL030-09

Valdujo PH, Camacho A, Recoder RS, Teixeira M, Ghellere JMB, Mott T, Nunes PMS, Nogueira C, Rodrigues MTU (2011) Anfíbios da Estação Ecológica Serra Geral do Tocantins, região do Jalapão, estados do Tocantins e Bahia. Biota Neotropica 11(1): 251-261. doi: 10.1590/S1676-06032011000100025

Verdade VK, Rodrigues MT, Pavan D (2009) Anfíbios Anuros da região da Estação Biológica do Alto da Serra de Paranapiacaba. In: Lopes MIMS, Kirizawa M, Melo MRF (Eds) Patrimônio da Reserva Biológica do Alto da Serra de Paranapiacaba. A antiga Estação Biológica do Alto da Serra. Secretaria do Meio Ambiente do Estado de São Paulo, São Paulo, 579-604.

Verdade VK, Valdujo PH, Carnaval AC, Schiesari L, Toledo LF, Mott T, Andrade GV, Eterovick PC, Menin M, Pimenta BVS, Nogueira C, Lisboa CS, De Paula CD, Silvano DL (2012) A leap further: the Brazilian amphibian conservation action plan. Alytes 29: 27-42. 
Xavier AL, Napoli MF (2011) Contribution of environmental variables to anuran community structure in the Caatinga Domain of Brazil. Phyllomedusa 10: 45-64. doi: 10.11606/ issn.2316-9079.v10i1p45-64

Young BE, Stuart SN, Chanson JS, Cox NA, Boucher TM (2004) Joyas que están desapareciendo: El estado de los anfibios en el Nuevo Mundo. NatureServe, Arlington, Virginia.

\section{Appendix I}

\section{Specimens examined}

Voucher specimens of the present study were deposited in the Museu de Zoologia da Universidade Estadual de Santa Cruz (MZUESC) at Universidade Estadual de Santa Cruz, municipality of Ilhéus, Bahia State, Brazil.

\section{AROMOBATIDAE}

Allobates olfersioides - MZUESC 8305.

BRACHYCEPHALIDAE

Brachycephalus pulex - MZUESC 8352, 8604-05, 10338-40.

Ischnocnema verrucosa - MZUESC 8323, 8464, 8829, 8993, 9090.

Ischnocnema sp. (gr. parva) - MZUESC 8057, 8059, 8234, 8312-13, 8366-68, 8460-61.

BUFONIDAE

Rhinella crucifer - MZUESC 8428-29, 8310.

Rhinella granulosa - MZUESC 9016.

Rhinella hoogmoedi - MZUESC 8380, 8601, 8904.

\section{CENTROLENIDAE}

Vitreorana eurygnatha - MZUESC 8308, 8373-75, 8441-42, 8520, 8911.

Vitreorana uranoscopa-MZUESC 8630, 9000-01.

\section{CRAUGASTORIDAE}

Haddadus binotatus - MZUESC 8045, 8327-28, 8421, 8435, 8437, 8611, 8906, 8909, 8927.

"Eleutherodactylus" bilineatus - MZUESC 8359, 8457, 8616-17, 8999.

Pristimantissp. 1 - MZUESC 8058, 8133, 8370-72, 8530, 8619-20, 8841-42, 9084-85.

Pristimantis sp. 2 - MZUESC 13272-73.

Pristimantis vinhai-MZUESC 8115, 8332, 8338, 8382, 8536-37, 8542, 8608, 8621, 8629.

\section{ELEUTHERODACTYLIDAE}

Adelophryne mucronatus - MZUESC 8458, 8068.

Adelophryne sp. - MZUESC 8049, 8358, 8447, 8838-40. 


\section{HEMIPHRACTIDAE}

Gastrotheca sp. - MZUESC 8827.

Gastrotheca pulchra-MZUESC 8120, 8314, 8463, 8828.

\section{HYLIDAE}

Aplastodiscus cf. weygoldti - MZUESC 8139, 8443, 8531-32, 8532, 8572, 8612-13, 8830-34.

Aplastodiscus ibirapitanga - MZUESC 8307, 8318, 8369, 8571, 8598-99, 8600, 8994, 9086.

Bokermannohyla circumdata - MZUESC 8360-61, 8439-40, 8526-29, 8618, 8826, 9074-76.

Bokermannohyla lucianae - MZUESC 8295-97, 8995.

Dendropsophus anceps - MZUESC 8419, 8857, 9048-49.

Dendropsophus bipunctatus - MZUESC 10332-34.

Dendropsophus branneri - MZUESC 8474-75.

Dendropsophus elegans - MZUESC 8849-50.

Dendropsophus giesleri - MZUESC 8590-92, 8855, 10335.

Dendropsophus haddadi - MZUESC 8362, 8476, 8568-69, 8577-82, 9087.

Dendropsophus novaisi - MZUESC 8565, 9025-27.

Dendropsophus minutus - MZUESC 8853, 9005.

Dendropsophus aff. oliveirai - MZUESC 8477-78, 8566-67.

Hypsiboas albomarginatus - MZUESC 8471, 8854, 8903.

Hypsiboas crepitans - MZUESC 8899.

Hypsiboas faber-MZUESC 8326, 8379.

Hypsiboas pombali - MZUESC 8302, 8378, 8631, 9018.

Hypsiboas semilineatus - MZUESC 13268-71.

Itapotihyla langsdorffi - MZUESC 9023-24.

Phasmahyla spectabilis - MZUESC 8150-51, 8294, 8298, 8303.

Phyllodytes wuchereri - MZUESC 8134, 8319, 9052.

Phyllodytes sp. 1 - MZUESC 8135-36, 8574.

Phyllomedusa burmeisteri - MZUESC 8309, 8470.

Phyllomedusa nordestina - MZUESC 9003-04.

Phyllomedusa rohdei - MZUESC 8846, 9015.

Scinax argyreornatus - MZUESC 8473, 8588-89.

Scinax juncae - MZUESC 8856, 13056-13068

Scinax x-signatus-MZUESC 8573, 8593-96.

Scinax eurydice - MZUESC 8041, 8113, 8432, 8467, 8603, 8847-48.

Scinax sp. 1 - MZUESC 8570, 8584-87.

Scinax sp. 2 (gr. rostratus) - MZUESC 8573.

Scinax strigilatus - MZUESC 8365, 8623-24, 8626, 8996, 9053, 9081, 9083.

Sphaenorhynchus prasinus - MZUESC 8597.

Trachycephalus mesophaeus - MZUESC 8315, 9019.

Trachycephalus nigromaculatus - MZUESC 8299, 8905. 


\section{HYLODIDAE}

Crossodactylus sp. - MZUESC 8056, 8121-22, 8521.

\section{LEPTODACTYLIDAE}

Adenomera cf. thomei-MZUESC 8138, 8317, 8364.

Leptodactylus cupreus - MZUESC 9041.

Leptodactylus fuscus - MZUESC 9002, 9017.

Leptodactylus cf. latrans - MZUESC 8915.

Leptodactylus mystaceus - MZUESC 8040.

Physalaemus camacan - MZUESC 9045-47.

Physalaemus erikae - MZUESC 8423, 9006, 9042-44.

\section{MICROHYLIDAE}

Chiasmocleis crucis - MZUESC 9028-29, 9031-34, 9036.

Stereocyclops histrio - MZUESC 9037-40.

Stereocyclops incrassatus - MZUESC 8422, 9020-22.

ODONTOPHRYNIDAE

Odontophrynus carvalhoi - MZUESC 8564.

Proceratophrys renalis - MZUESC 10341.

Proceratophrys schirchi - MZUESC 8152, 8300, 8468-69, 8602, 10342-45.

PIPIDAE

Pipa carvalhoi - MZUESC 7360.

\section{SIPHONOPIDAE}

Siphonops annulatus - MZUESC 8376, 8459, 8913, 9056, 9070. 